\title{
Long-Acting Growth Hormone Preparations and Their Use in Children with Growth Hormone Deficiency
}

\author{
Valentina Pampanini ${ }^{a}$ b $\quad$ Annalisa Deodati ${ }^{a} \quad$ Elena Inzaghi ${ }^{c}$ \\ Stefano Cianfarani ${ }^{\mathrm{a}, \mathrm{b}} \mathrm{d}$ \\ aDipartimento Pediatrico Universitario Ospedaliero, IRCCS "Bambino Gesù" Children's Hospital, Rome, Italy; \\ bKarolinska Institutet, Department of Women's and Children's Health, Stockholm, Sweden; 'Pediatric Emergency \\ Department and General Pediatrics, IRCCS "Bambino Gesù" Children's Hospital, Rome, Italy; dDepartment of \\ Systems Medicine, University of Rome Tor Vergata, Rome, Italy
}

\section{Keywords}

Growth hormone - Long-acting growth hormone •

Growth · Short stature - Growth hormone deficiency ·

Growth hormone therapy

\begin{abstract}
Background: Daily recombinant human growth hormone (rhGH) is approved and marketed worldwide to treat children and adults with $\mathrm{GH}$ deficiency and other conditions. Efficacy of rhGH therapy is influenced by several variables. Drop of treatment adherence over time has been recognized as a cause of reduced rhGH efficacy and has driven considerable efforts from pharmaceutical companies and scientists to develop long-acting rhGH (LAGH) formulations in order to relieve patients and their families from the burden of daily injections. Summary: Different technologies to manipulate drug release have been produced allowing weekly, biweekly, or monthly rhGH administration. The LAGH formulations developed at present have demonstrated a comparable or even higher efficacy as compared with daily rhGH in most of the cases and no major safety issues in phase 3 studies. A greater incidence of injection-site reactions has been reported but mainly of mild and transient nature. Key Messages:
\end{abstract}

Karger@karger.com

(c) 2022 S. Karger AG, Basel

www.karger.com/hrp

Karger"
Despite LAGH analogs appearing promising, potential drawbacks still need to be addressed. Long-term consequences of nonphysiological GH profile and its consequences on metabolism and risk of cancer, optimal therapeutic monitoring, immunogenicity of LAGH molecules, and potential novel side effects related to the technologies used to develop these molecules are among the major concerns that require answers from long-term surveillance. Finally, increased acceptance of LAGH formulations from patients and their caregivers is yet to be demonstrated and cost-effectiveness evaluated consequently.

(c) 2022 S. Karger AG, Basel

\section{Introduction}

Since 1985, recombinant human growth hormone (rhGH) has been used for the treatment of GH deficiency (GHD). Efficacy and safety of rhGH administered daily have been widely documented [1-6]. As reports on clinical outcomes of patients treated with daily rhGH multiplied over the years, low adherence to the therapy emerged as a significant cause of suboptimal therapeutic response [7]. Daily injections demand efforts from patients and 
caregivers that can result in a decline of adherence over the years. Long-acting GH (LAGH) formulations have been proposed as a potential alternative to reduce the burden on children and their families by decreasing the injection frequency, ultimately improving treatment outcomes. Starting from 1999, when the first LAGH was approved and commercialized, several pharmaceutical companies have developed long-acting formulations that can be administered weekly, biweekly, or monthly (Table 1). Methodological strategies to extend GH pharmacokinetic curves include manipulation of drug release from subcutaneous depots and manipulation of in vivo clearance from the circulation.

Sustained drug release from depots has been developed by: (a) dispersion of rhGH in polymeric materials to form microspheres; (b) encapsulation of rhGH in sodium hyaluronate microparticles suspended in medium-chain triglycerides; (c) transient conjugation (TransCon) technology; (d) supercritical carbon dioxide; and (e) protein crystallization. Delayed clearance has been obtained by: (a) addition of polyethylene glycol (PEG) moieties (i.e., PEGylation) that increase the size of rhGH molecules, thereby reducing renal clearance and immunogenicity; (b) conjugation of rhGH to a fatty acid chain that enhances noncovalent binding to serum albumin resulting in prolonged half-life; and (c) rhGH fusion with the Fc domain of an immunoglobulin $\mathrm{G}$ or human serum albumin or other proteins also extending the therapeutic compound persistence within the circulation. In this work, we will review the current status of LAGH analogs in terms of drug development and data on efficacy and safety in children with GHD.

\section{Efficacy and Safety Considerations for LAGH Formulations}

A variety of LAGH formulations are being investigated in clinical trials, and as the availability of these formulations is expected to increase, issues on efficacy, safety, and monitoring remain to be addressed. Once- or biweekly administration of rhGH unavoidably results in the loss of the pulsatile pattern that characterizes GH secretion. Nonphysiological GH and IGF-I serum profiles might have a long-term impact on glucose and fat metabolism as well as on body composition. Furthermore, doses needed to reach clinical efficacy could result in supraphysiological IGF-I serum levels, with possible implications for cancer risk in the long term. Differences in pharmacokinetic and pharmacodynamic profiles of
LAGH analogs may impact on both the efficacy and safety of these preparations in a way that is unique to each preparation. Additional molecules and drug manipulations used to create LAGH analogs might originate unknown side effects per se. Furthermore, the nonreversibility of GH exposure could be a clinically relevant problem in case of onset of serious adverse events (i.e., benign intracranial hypertension). In terms of efficacy, manipulation of the GH molecule to create LAGH formulations might result in increased immunogenicity and formation of neutralizing antibodies with subsequent reduced drug bioavailability and efficacy. Another potential efficacy pitfall might arise in very young children with severe GHD because of the risk of hypoglycemia when the lowest concentration of rhGH is reached before the next dose. Finally, for analogs created by increasing the molecule size (e.g., PEGylated LAGH), penetrance into target tissues might be suboptimal.

\section{Classes of LAGH Formulations}

\section{Depot Formulations}

Nutropin Depot ${ }^{\circledR}$ was the first LAGH for injection every 2 weeks, marketed in 1999 and based on microsphere technology. Silverman et al. [8] reported data on efficacy and safety after 2 years of treatment in 56 prepubertal children with documented GHD. The growth rate in the cohort was $8.2 \pm 1.8 \mathrm{~cm} /$ year in the first year and $7.0 \pm 1.5$ $\mathrm{cm} /$ year in the second year of treatment, significantly lower than the growth rate after daily rhGH injections in historical controls with matched baseline characteristics. Height gain after 24 months was $1.0 \pm 0.5$ standard deviation score (SDS), $p<0.001$ versus baseline but significantly lower when compared with historical controls; no major side effects were observed, although pain and nodules in the injection site were commonly reported [8]. The drug production was discontinued in 2004.

Eutropin Plus $^{\mathrm{TM}}$ (LB03002) is a once-weekly rhGH encapsulated in sodium hyaluronate microparticles; once administered subcutaneously, the microparticles are degraded by tissue hyaluronidase allowing sustained GH release. It was first marketed in South Korea for children with GHD. In 2012, Peter et al. [9] reported the efficacy and safety of LB03002 in GHD prepubertal children after a 3-year randomized, controlled, multicenter, phase $2 / 3$ study involving six European centers. Participants were randomized into four groups ( $N=13$ subjects per group) treated with increasing doses of the LAGH $(0.2 \mathrm{mg} / \mathrm{kg} /$ week, $0.5 \mathrm{mg} / \mathrm{kg} /$ week, and $0.7 \mathrm{mg} / \mathrm{kg} /$ week) or daily in- 


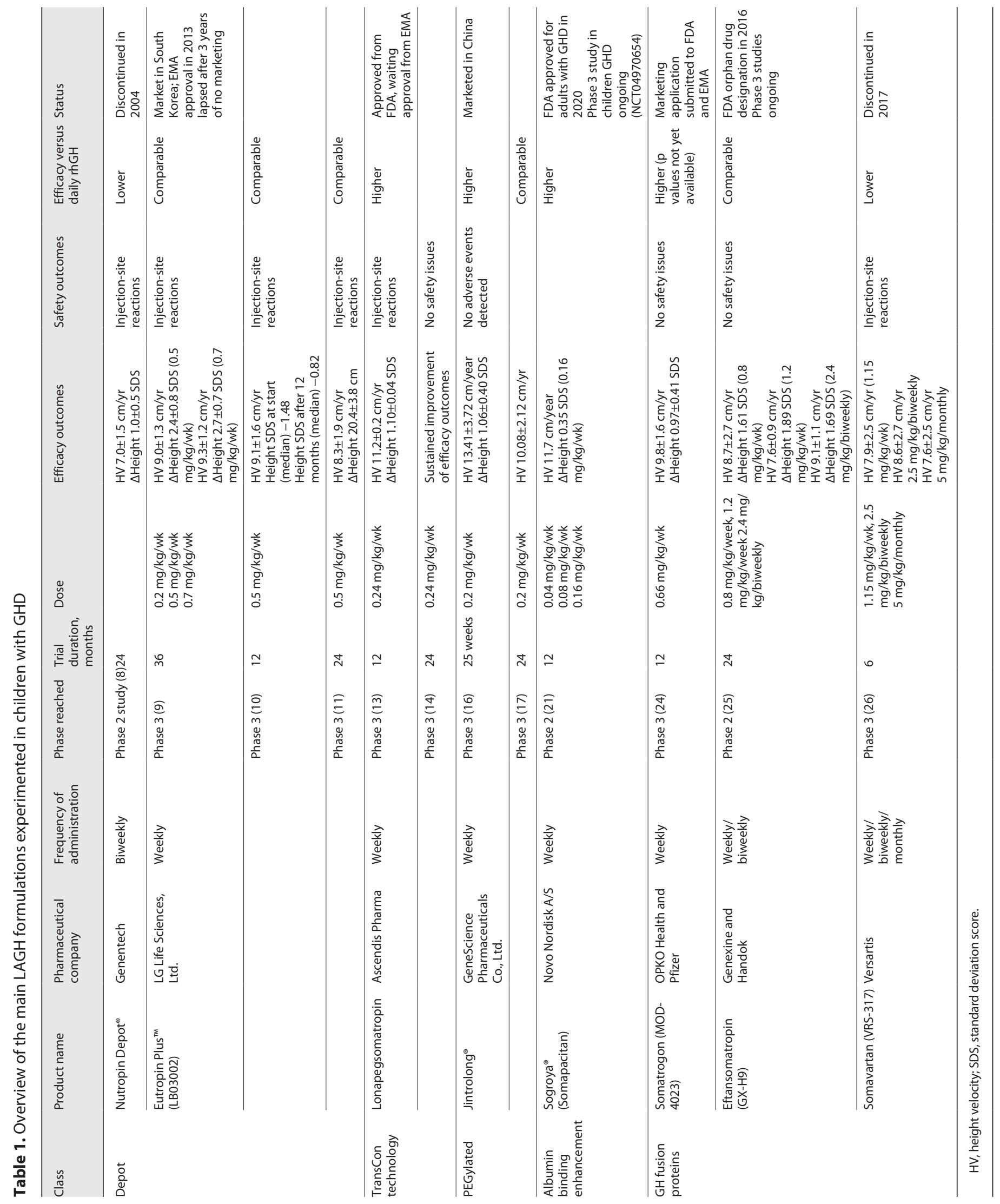


jections $(0.03 \mathrm{mg} / \mathrm{kg} / \mathrm{day})$. Doses of 0.5 and $0.7 \mathrm{mg} / \mathrm{kg} /$ week were as effective as daily GH in terms of height gain and IGF-I increase, and the safety profile was comparable to that of daily $\mathrm{GH}[9]$.

The following year a Korean study confirmed a comparable efficacy in terms of 1-year growth rate between weekly LB03022 at the dose of $0.5 \mathrm{mg} / \mathrm{kg} /$ day and daily $\mathrm{rhGH}$ injections $(0.03 \mathrm{mg} / \mathrm{kg} /$ day) [10]. Another randomized, controlled, multicenter, phase 3 trial was conducted between 2011 and 2013. Weekly LB03002 was administered to 91 GHD children and daily rhGH to 87 GHD children for 1 year; in the following 12 months, all children were treated with once-weekly LB03002, again confirming comparable growth response between weekly and daily formulation with an increase in injection-site reactions for long-acting formulation that were mild to moderate in the majority of cases [11]. Despite European Medicines Agency (EMA) approval of Eutropin Plus ${ }^{\mathrm{TM}}$ in 2013, the drug was not commercialized in Europe, causing the authorization to lapse after 3 years.

\section{TransCon Technology}

TransCon GH from Ascendis Pharma (Lonapegsomatropin) is a ternary complex formed between an unmodified rhGH molecule, an inert carrier that shields the drug, and a temporary linker. After subcutaneous injection, $\mathrm{pH}$ and body temperature induce the linker selfcleaving, thereby initiating the release of the rhGH at a predetermined rate.

Phase 2 trial in children with GHD was published by Chatelain et al. [12] in 2017 and revealed comparable efficacy and safety of the LAGH analog when compared to daily rhGH. Three phase 3 trials followed, the heiGHt trial (NCT02781727), the fliGHt trial (NCT03305016), and the enliGHten trial (NCT03344458). The pivotal 52-week heiGHt trial included 161 treatment-naïve GHD children from 73 sites across 15 countries [13]. Weekly administration of Lonapegsomatropin induced a greater height velocity (HV) after 12 months than with daily rhGH and a comparable increase in height SDS. Safety and tolerability were similar between the two treatment groups, and no increased immunogenicity of the LAGH compound was detected. IGF-I levels peaked $48 \mathrm{~h}$ after Lonapegsomatropin and returned to predose levels by the end of the dose interval. A steady state of IGF-I concentration was reached at week 13 of treatment, earlier than with daily rhGH. Estimated average IGF-1 SDS was higher throughout the trial, rarely exceeding +2.0 SDS, consistently with the greater HV. The fliGHt trial confirmed the efficacy and safety outcomes after switching from daily rhGH to
Lonapegsomatropin for 26 weeks [14]. A total of 298 subjects continued thereafter into the long-term extension enliGHten trial that demonstrated sustained improvement of height SDS and a good safety profile after 2 years of treatment [14]. Data have been submitted to Food and Drug Administration (FDA) and EMA in early 2021 resulting in FDA approval of TransCon hGH, which is currently marketed in the USA, whereas a final EMA decision is expected by early 2022 .

\section{PEGylated Formulations}

The only PEGylated rhGH currently present on the market is once-weekly Jintrolong, exclusively available in China. Drug's clinical testing started with a phase I trial performed in 2015 on 12 children with GHD [15], followed by phase 2 and 3 multicentric trials involving six Chinese hospitals [16]. Treatment-naïve GHD children were treated for 25 weeks; in phase 2 study, 97 patients were randomized into three arms of treatment, balanced for sex, age, midparental height, and clinical characteristics (including height SDS, IGF-I levels, HV, and percentage of multiple pituitary hormone deficiencies). PEG-rh$\mathrm{GH}$ was administered at the doses of $0.1 \mathrm{mg} / \mathrm{kg} /$ week and $0.2 \mathrm{mg} / \mathrm{kg} /$ week and compared to daily $\mathrm{rhGH}$ at the dose of $0.25 \mathrm{mg} / \mathrm{kg} /$ week. HV, $\Delta$ height SDS, and IGF-I levels were increased in the three groups of treatment with a significant greater effect of the higher dose of PEG-rhGH and the daily rhGH versus PEG-rhGH at lower dose. Adverse events were comparable between the PEG-rhGH and the daily rhGH groups. The phase 3 study included a larger cohort of patients $(n=343)$ randomized into two groups (PEG-rhGH at the dose of $0.2 \mathrm{mg} / \mathrm{kg} /$ week vs. daily $\mathrm{rhGH}$ at the dose of $0.25 \mathrm{mg} / \mathrm{kg} /$ week) and demonstrated a significant greater increase of HV, height SDS, and IGF-I levels after 25 weeks of treatment with Jintrolong, as compared with daily rhGH. In 2019, other authors in China published single-center prospective data after two years of treatment of GHD children with Jintrolong, confirming previous findings on efficacy and safety [17]. Other attempts to develop PEGylated formulations have not been successful in providing sufficient efficacy and/or safety profiles. ARX201 from Ambryx and PHA-794428 from Pfizer have no longer been developed after evidence of PEG accumulation in the ependymal cell of the choroid plexus in primates and high rates of lipoatrophy in the injection site, respectively. Novo Nordisk also ended the development of its weekly PEG-rhGH named NNC126-0083 in 2011 after a study from a Belgian group reported unsatisfactory IGF-I profile after administration of the weekly PEG-rhGH. 


\section{Noncovalent Albumin Binding Enhancement}

Somapacitan is a once-weekly formulation in which rhGH is conjugated to a fatty acid that can bind serum albumin slowing drug elimination. Efficacy and safety of Somapacitan have been widely documented both in healthy adults [18] and in adult patients with GHD [19]. In 2017, Battelino et al. [20] reported the pharmacodynamics and pharmacokinetics of single doses of Somapacitan in children with GHD: drug's serum concentrations and increase in IGF-I and IGFBP3 levels were measured after administration of escalating doses $(0.02,0.04$, 0.08 , and $0.16 \mathrm{mg} / \mathrm{kg}$ ) of the LAGH and compared with once-daily rhGH at the dose of $0.03 \mathrm{mg} / \mathrm{kg}$. All doses were well tolerated, and all doses except the lowest one $(0.02$ $\mathrm{mg} / \mathrm{kg}$ ) induced an IGF-I increase.

Response was comparable to the IGF-I response generated by daily rhGH, opening the avenue to multiple dose phase I trials. However, since IGF-I accumulation could not be assessed in the single-doses study, Juul et al. [21] performed a pooled modeling analysis of data from the three existing phase I randomized trials performed in healthy adults [18], adults with GHD [19], and children with GHD [20] in order to predict the pharmacokinetic (maximal concentration, $C_{\max }$; half-life, $\mathrm{t}_{1 / 2}$; area under the curve) and pharmacodynamic profiles (IGF-I and IGFBP3 increase) after multiple doses.

Results of the study confirmed a good response in terms of IGF-I increase to weekly administration of Somapacitan with negligible accumulation of the drug. This study was followed by REAL 3 phase 2 trial that compared 3 different doses of Somapacitan with daily rhGH in 59 treatment-naïve children with GHD [22]. After 26 weeks of treatment, HV increased in a dosedependent manner; medium and higher doses of Somapacitan $(0.08 \mathrm{mg} / \mathrm{kg} /$ week and $0.16 \mathrm{mg} / \mathrm{kg} /$ week $)$ produced an increase in $\mathrm{HV}$ as with daily rhGH. Extension of the trial for 26 weeks more (total 52 weeks) showed a sustained HV and a change in height SDS from baseline that were significantly greater than with daily $\mathrm{GH}$ when $0.16 \mathrm{mg} / \mathrm{kg} /$ week Somapacitan was used. Doses of $0.08 \mathrm{mg} / \mathrm{kg} /$ week produced a similar increase of IGF-I SDS as compared with daily rhGH. A significantly greater increase of IGF-I SDS after 26 and 52 weeks was observed in response to doses of Somapacitan of 0.16 $\mathrm{mg} / \mathrm{kg} /$ week than with daily rhGH. Administration of $0.16 \mathrm{mg} / \mathrm{kg} /$ week was then prolonged to test safety for 208 weeks. No clinically significant safety issues were reported, and the local tolerability in terms of injectionsite reactions was not affected at any dose. A phase 3 trial started in China in July 2021 and is presently re-

Long-Acting Growth Hormone cruiting GHD children (NCT04970654) to evaluate the efficacy and safety of $0.16 \mathrm{mg} / \mathrm{kg} /$ week Somapacitan for 52 weeks.

\section{GH Fusion Proteins}

Three different LAGH produced by fusing proteins to rhGH molecule to prolong the half-life and reduce immunogenicity reached phase 3 study stage in children with GHD. Somatrogon (MOD-4023) is an analog created by OPKO Health and Pfizer by fusing three copies of a C-terminal peptide of human chorionic gonadotropin $\beta$-subunit to the coding sequence of rhGH [23]. Data from two clinical trials in healthy adults (unpublished) and adults with GHD [24] were used to infer pharmacokinetic and pharmacodynamic models for use in children. Phase 2 study conducted by Zelinska et al. [25] assessed the efficacy, safety, local tolerability, pharmacokinetics, and pharmacodynamics of weekly MOD-4023 administered for 12 months at three different doses and compared with daily rhGH.

The highest dose tested $(0.66 \mathrm{mg} / \mathrm{kg} /$ week $)$ proved to have the same efficacy in terms of $\mathrm{HV}$ and $\Delta$ height SDS as daily rhGH. The incidence rate of adverse events was similar between the long-acting analog and daily rhGH, and no major issues in local tolerability were reported. MOD-4023 estimated half-life was 5- to 10-fold longer than daily rhGH and produced an increase in IGF-I and IGFBP3 SDS that was dose-dependent, below the safety threshold of +2 SDS, and comparable to that induced by daily rhGH with the medium and higher doses used ( 0.48 and $0.66 \mathrm{mg} / \mathrm{kg} /$ week).

Results of a phase 3 study on efficacy and safety of Somatogron in children have been reported in August 2021 [26]. GHD children were treated for 12 months. In the first 6 weeks, a pharmacokinetic and pharmacodynamic sampling was performed by treating patients with escalating doses $(0.25 \mathrm{mg} / \mathrm{kg} /$ week, $0.48 \mathrm{mg} / \mathrm{kg} /$ week, and 0.66 $\mathrm{mg} / \mathrm{kg} /$ week), each administered for 2 weeks. For the remaining time (46 weeks), patients were treated with either Somatogron at $0.66 \mathrm{mg} / \mathrm{kg} /$ week or daily rhGH. The phase 3 study confirmed the noninferiority of MOD-4023 as compared to daily rhGH in terms of clinical outcomes, confirmed good tolerability and safety, and reported improved adherence and burden perceived by patients. Marketing application has been submitted to the FDA in January 2021 and to the EMA. A decision from the European Commission is expected in 2022.

Another LAGH analog with seemingly promising preliminary results in pediatric GHD is GX-H9 from Genexine and Handok. GX-H9 is a rhGH fused to the Fc portions of $\operatorname{IgD}$ and immunoglobulin $\mathrm{G} 4$. Phase 2 dose-find- 
Table 2. Summary of issues that remain to be addressed for use of LAGH formulations in children GHD

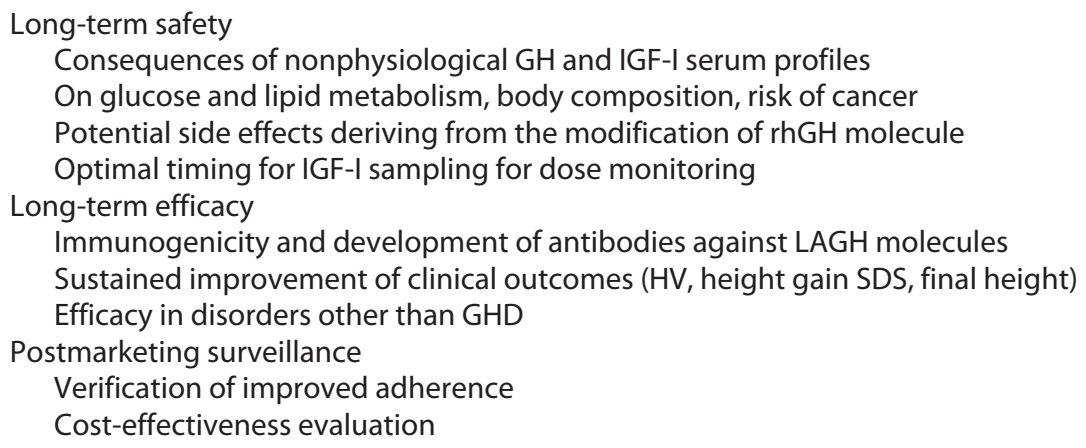

ing trial in children with GHD reported a comparable efficacy and safety of GX-H9 to daily rhGH [27]. Finally, Somavartan (VRS-317) from Versartis reached a phase 3 study level in children with GHD but was no longer developed after the study proved it to be inferior to daily rhGH in increasing HV and height SDS [28].

\section{Concluding Remarks}

LAGH preparations have been developed with the aim of improving the adherence of patients to therapy and, ultimately, the clinical outcomes. The LAGH analogs experimented up to phase 3 in clinical trials, so far, have not shown substantial differences in terms of short-term clinical outcomes and safety compared to daily rhGH. However, increased cardio- and cerebrovascular mortality after long-term treatment with daily rhGH has recently been reported, thus prompting the need of further longterm surveillance for both daily and LAGH preparations [29]. Though LAGH is expected to share most if not all the risks of daily rhGH, there may be additional risks depending on the composition, pharmacokinetics, and pharmacodynamics of the different LAGH formulations (Table 2). First, the use of LAGH products obtained by modifications of GH molecule may induce the development of anti-GH antibodies which, in case of neutralizing properties, may affect the response to therapy. Second, LAGH-based treatments are far from mimicking the physiology of GH secretion. The daily spontaneous pulsatile GH secretion patterns as well as the nocturnal GH peak (mimicking the natural sleep time peak GH secretion) induced by daily rhGH injected at bedtime are not fulfilled by LAGH. Whether the unnatural LAGH pharmacokinetics may have metabolic consequences in the long term should be investigated by longitudinal studies. Finally, the profile of the IGF-I response to each LAGH should be characterized. High circulating levels of IGF-I have been associated with increased risk of certain cancers. Though there is no evidence at the moment of specific risks associated with transient elevations of IGF-I levels, keeping IGF-I within the normal range should be considered a goal to be achieved when using LAGH as well as rhGH. In this context, the optimal timing of IGFI measurement for dose adjustments varies among the different formulations and needs to be defined through the development of pharmacodynamic modeling specific to each LAGH analog in order to monitor the safety of treatment.

Last but not least, there is no evidence yet that the use of LAGH improves the adherence to therapy and, consequently, the clinical outcome in the long term. Postmarketing surveillance registries together with observational studies will be crucial to assess efficacy, safety, tolerability, and cost-effectiveness of LAGH-based therapies.

\section{Conflict of Interest Statement}

S.C. is an editor in chief of Hormone Research in Paediatrics. The other authors have no conflicts of interest to declare.

\section{Funding Sources}

The authors did not receive any funding.

\section{Author Contributions}

V.P. reviewed the literature and initiated this review. A.D. made substantial contribution to the design of the presented review. E.I. made substantial contribution to the concept and revision of the presented review. S.C. took the lead in writing the text and revised the tables and the text. All authors read and approved the manuscript. 


\section{References}

1 Ranke MB. Short and long-term effects of growth hormone in children and adolescents with GH deficiency. Front Endocrinol. 2021; 12:720419.

2 Savendahl L, Polak M, Backeljauw P, Blair JC, Miller BS, Rohrer TR, et al. Long-term safety of growth hormone treatment in childhood: two large observational studies: nordinet IOS and answer. J Clin Endocrinol Metab. 2021; 106(6):1728-41.

3 Tidblad A, Bottai M, Kieler H, AlbertssonWikland K, Sa vendahl L. Association of childhood growth hormone treatment with long-term cardiovascular morbidity. JAMA Pediatr. 2021;175(2):e205199.

4 Swerdlow AJ, Cooke R, Albertsson-Wikland K, Borgstrom B, Butler G, Cianfarani S, et al. Description of the SAGhE Cohort: a Large European study of mortality and cancer incidence risks after childhood treatment with recombinant growth hormone. Horm Res Paediatr. 2015;84(3):172-83.

5 Savendahl L, Polak M, Backeljauw P, Blair J, Miller BS, Rohrer TR, et al. Treatment of children with GH in the United States and Europe: long-term follow-up from $\operatorname{NordiNet(R)}$ IOS and answer program. J Clin Endocrinol Metab. 2019;104(10):4730-42.

6 Deodati A, Cianfarani S. Impact of growth hormone therapy on adult height of children with idiopathic short stature: systematic review. BMJ. 2011;342:c7157.

7 Fisher BG, Acerini CL. Understanding the growth hormone therapy adherence paradigm: a systematic review. Horm Res Paediatr. 2013;79(4):189-96.

8 Silverman BL, Blethen SL, Reiter EO, Attie KM, Neuwirth RB, Ford KM. A long-acting human growth hormone (Nutropin Depot): efficacy and safety following two years of treatment in children with growth hormone deficiency. J Pediatr Endocrinol Metab. 2002; 15(Suppl 2):715-22.

9 Peter F, Bidlingmaier M, Savoy C, Ji HJ, Saenger PH. Three-year efficacy and safety of LB03002, a once-weekly sustained-release growth hormone $(\mathrm{GH})$ preparation, in prepubertal children with GH deficiency (GHD). J Clin Endocrinol Metab. 2012;97(2):400-7.

10 Hwang JS, Lee HS, Chung WY, Han HS, Jin DK, Kim HS, et al. Efficacy and safety of LB03002, a once-weekly sustained-release human GH for 12-month treatment in Korean children with GH deficiency. Eur J Endocrinol. 2013;169(2):179-85.

11 Khadilkar V, Radjuk KA, Bolshova E, Khadgawat R, El Kholy M, Desai M, et al. 24-Month use of once-weekly GH, LB03002, in prepubertal children with GH deficiency. J Clin Endocrinol Metab. 2014;99(1):126-32.

12 Chatelain P, Malievskiy O, Radziuk K, Senatorova G, Abdou MO, Vlachopapadopoulou E, et al. A Randomized Phase 2 study of longacting TransCon GH versus daily GH in childhood GH deficiency. J Clin Endocrinol Metab. 2017;102(5):1673-82.

13 Thornton PS, Maniatis AK, Aghajanova E, Chertok E, Vlachopapadopoulou E, Lin Z, et al. Weekly lonapegsomatropin in treatmentnaive children with growth hormone deficiency: the phase 3 height trial. J Clin Endocrinol Metab. 2021.

14 Maniatis AK, Nadgir UM, Hofman P, Saenger $\mathrm{P}$, Chertok E, Aghajanova EM, et al. Efficacy and safety of up to 2 years of treatment with TransCon hGH (Lonapegsomatropin) in treatment-naïve and treatment-experienced children with growth hormone deficiency. J Endocr Soc. 2021:5.

15 Hou L, Chen ZH, Liu D, Cheng YG, Luo XP. Comparative pharmacokinetics and pharmacodynamics of a PEGylated recombinant human growth hormone and daily recombinant human growth hormone in growth hormonedeficient children. Drug Des Devel Ther. 2016;10:13-21.

16 Luo X, Hou L, Liang L, Dong G, Shen S, Zhao $Z$, et al. Long-acting PEGylated recombinant human growth hormone (Jintrolong) for children with growth hormone deficiency: phase II and phase III multicenter, randomized studies. Eur J Endocrinol. 2017;177(2):195205.

17 Qiao Y, Wang Z, Han J, Li G. Use of PEGylated recombinant human growth hormone in Chinese children with growth hormone deficiency: a 24-month follow-up study. Int J Endocrinol. 2019;2019:1438723.

18 Rasmussen MH, Olsen MW, Alifrangis L, Klim S, Suntum M. A reversible albuminbinding growth hormone derivative is well tolerated and possesses a potential onceweekly treatment profile. J Clin Endocrinol Metab. 2014;99(10):E1819-29.

19 Rasmussen MH, Janukonyte J, Klose M, Marina $\mathrm{D}$, Tanvig $\mathrm{M}$, Nielsen LF, et al. Reversible albumin-binding $\mathrm{GH}$ possesses a potential once-weekly treatment profile in adult growth hormone deficiency. J Clin Endocrinol Metab. 2016;101(3):988-98.

20 Battelino T, Rasmussen MH, De Schepper J, Zuckerman-Levin N, Gucev Z, Savendahl L, et al. Somapacitan, a once-weekly reversible albumin-binding GH derivative, in children with GH deficiency: a randomized dose-escalation trial. Clin Endocrinol. 2017;87(4):3508.

21 Juul RV, Rasmussen MH, Agersø H, Overgaard RV. Pharmacokinetics and pharmacodynamics of once-weekly somapacitan in children and adults: supporting dosing rationales with a model-based analysis of three phase I trials. Clin Pharmacokinet. 2019; 58(1):63-75.

22 Savendahl L, Battelino T, Brod M, Hojby Rasmussen M, Horikawa R, Juul RV, et al. Onceweekly somapacitan versus daily GH in children with GH deficiency: results from a randomized phase 2 trial. J Clin Endocrinol Metab. 2020;105(4):e1847.

23 Hershkovitz O, Bar-Ilan A, Guy R, Felikman $\mathrm{Y}$, Moschcovich L, Hwa V, et al. Vitro and in vivo characterization of MOD-4023, a longacting carboxy-terminal peptide (CTP)-modified human growth hormone. Mol Pharm. 2016;13(2):631-9.

24 Strasburger CJ, Vanuga P, Payer J, Pfeifer M, Popovic V, Bajnok L, et al. MOD-4023, a long-acting carboxy-terminal peptide-modified human growth hormone: results of a Phase 2 study in growth hormone-deficient adults. Eur J Endocrinol. 2017;176(3):283-94.

25 Zelinska N, Iotova V, Skorodok J, Malievsky O, Peterkova V, Samsonova L, et al. Long-acting C-terminal peptide-modified $\mathrm{hGH}$ (MOD-4023): results of a safety and dosefinding study in Ghd children. J Clin Endocrinol Metab. 2017;102(5):1578. https://doi. org/10.1210/jc.2016-3547.

26 https: //clinicaltrials.gov/ct2/show/results/ NCT03874013?term $=$ mod-4023\& draw $=2 \&$ rank=1.

27 Malievskiy OMA, Zelinska N, Bolshova E, Senatorova G, Oroszlán G, Skorodok J, et al. SAT-LB15 24-month efficacy and safety of once weekly and every other week administration of GX-H9, hybrid FC-fused long-acting human growth hormone: a phase 2 study in children with growth hormone deficiency. J Endocr Soc. 2020;4:1.

28 Moore WV, Nguyen HJ, Kletter GB, Miller $\mathrm{BS}$, Rogers D, Ng D, et al. A randomized safety and efficacy study of somavaratan (VRS317), a long-acting rhGH, in pediatric growth hormone deficiency. J Clin Endocrinol Metab. 2016;101(3):1091-7.

29 Savendahl L, Cooke R, Tidblad A, Beckers D, Butler G, Cianfarani S, et al. Long-term mortality after childhood growth hormone treatment: the SAGhE cohort study. Lancet Diabetes Endocrinol. 2020;8(8):683-92. 